\title{
On the tracking performance of least squares MIMO channel estimation in rician fading
}

\section{Irma Zakia ${ }^{\mathrm{a})}$, Suhartono Tjondronegoro, Iskandar, and Adit Kurniawan}

School of Electrical Engineering and Informatics, Institut Teknologi Bandung, Bandung, Indonesia

a)irma.zakia@students.itb.ac.id

\begin{abstract}
The tracking Mean Square Error (MSE) of the least squares (LS) Multiple Input Multiple Output (MIMO) channel estimation in rician flat fading channel is derived in closed-form, which is then verified by Monte Carlo simulation. The channel accomodates applications where the elevation angle between the transmitter and the receiver dictates the doppler shift of the Line of Sight (LOS) component, as encountered in communications from satellite or High Altitude Platform (HAP) to a moving mobile. A higher tracking loss is revealed as the mobile travels to a lower elevation angle.
\end{abstract}

Keywords: rician fading, elevation angle, least squares (LS), channel tracking, doppler shift

Classification: Wireless Communication Technologies

\section{References}

[1] Iskandar and S. Shimamoto, "Channel characterization and performance evaluation of mobile communication employing stratospheric platforms," IEICE Trans. Commun., vol. E89-B, no. 3, pp. 937-944, March 2006.

[2] J. Holis and P. Pechac, "Elevation dependent shadowing model for mobile communications via high altitude platforms in built-up areas," IEEE Trans. Antennas Propag., vol. 56, no. 4, pp. 1078-1084, April 2008.

[3] E. Falletti, F. Sellone, C. Spillard, and D. Grace, "A transmit and receive multi-antenna channel model and simulator for communications from high altitude platforms," International Journal of Wireless Information Networks, vol. 13, no. 1, pp. 59-75, Jan. 2006.

[4] M. Paetzold, U. Killat, and F. Laue, "An extended suzuki model for land mobile satellite channels and its statistical properties," IEEE Trans. Veh. Technol., vol. 47, no. 2, pp. 617-630, May 1998.

[5] E. Karami, "Tracking performance of least squares MIMO channel estimation algorithm," IEEE Trans. Commun., vol. 55, no. 11, pp. 2201-2208, Nov. 2007.

[6] C. Komninakis, C. Fragouli, A. Sayed, and R. D. Wesel, "Multi-Input multi-output fading channel tracking and equalization using kalman estimation," IEEE Trans. Signal Process., vol. 50, no. 5, pp. 1065-1076, May 2002. 


\section{Introduction}

In the context of satellite or HAP, the elevation angle between the transmitter and receiver is an important parameter in analyzing their performance. The literatures in [1] and [2] model the elevation angle dependency on $K$ factor and shadowing respectively. For communications to a moving user, the elevation angle dictates the doppler shift of the LOS component, whereas scatterers surrounding the mobile introduce doppler spread of the nonLOS (NLOS) component. As such, the channel is time varying rician distributed [3, 4].

If only the LOS is of concern, acquiring the doppler shift is accomplished by frequency tracking algorithms. In contrast, the $K$ factor varies from 0 $20 \mathrm{~dB}$, depending on the elevation angle [1]. As such, it is required to track the overall rician channel. Examples of channel tracking algorithm include the LS for rayleigh fading [5] and Kalman filter for rician fading with constant LOS [6]. Since the LS algorithm is independent of the underlying channel model, extending it to track the rician channel for which the LOS is affected by elevation angle as assumed here, is straightforward. It is therefore of interest to analyze the impact of elevation angle on the LS tracking performance.

In light of a previous work on tracking performance of LS MIMO channel estimation in rayleigh fading [5], we generalized the closed-form tracking MSE to MIMO rician flat fading channel. The derivation is a function of the $K$ factor, elevation angle, forgetting factor, number of antenna transmit, normalized doppler, Signal to Noise Ratio (SNR), and training sequence index, which is then verified by simulation. The approach taken follows closely [5]. However, it is observed that the statistical expectation matrix contributed by the LOS channel results in an additional hurdle to the analysis, for which by numerical evaluation, a very good analytical expression is found. A higher tracking loss is revealed as the mobile travels to a lower elevation angle.

\section{System model}

With $M$ antenna transmit and $N$ antenna receive,

$$
\boldsymbol{y}[n]=\boldsymbol{H}[n] \boldsymbol{x}[n]+\boldsymbol{v}[n]
$$

where $\boldsymbol{y}[n] \in \mathbb{C}^{N, 1}, \boldsymbol{H}[n] \in \mathbb{C}^{N, M}, \boldsymbol{x}[n] \in \mathbb{C}^{M, 1}$, and $\boldsymbol{v}[n] \in \mathbb{C}^{N, 1}$ denote the discrete-time MIMO received vector at time $n$, the MIMO rician flat fading channel matrix, the transmitted symbol vector generated from Phase Shift Keying (PSK) constellation [5], and the Additive White Gaussian Noise (AWGN) vector respectively. The noise $\boldsymbol{v}[n]$ is zero mean i.i.d. gaussian distributed with variance $\sigma_{v}^{2}$. By defining $\beta=\frac{K}{K+1}, 0 \leq \beta \leq 1$,

$$
\boldsymbol{H}[n]=\sqrt{\beta} \boldsymbol{H}_{\mathrm{LOS}}[n]+\sqrt{1-\beta} \boldsymbol{H}_{\mathrm{NLOS}}[n]
$$

where $K, \boldsymbol{H}_{\mathrm{LOS}}[n] \in \mathbb{C}^{N, M}$ and $\boldsymbol{H}_{\mathrm{NLOS}}[n] \in \mathbb{C}^{N, M}$ denote the rician factor, the LOS channel matrix, and the NLOS channel matrix respectively. All elements in $\boldsymbol{H}_{\mathrm{LOS}}[n], \boldsymbol{H}_{\mathrm{NLOS}}[n]$, and consequently $\boldsymbol{H}[n]$, have unit power.

Assuming a stationary transmitter, the LOS channel matrix is [3]

$$
\boldsymbol{H}_{\mathrm{LOS}}[n]=e^{j(\kappa n+\phi)} \boldsymbol{a}_{\mathrm{rx}}(\theta) \boldsymbol{a}_{\mathrm{tx}}^{\mathrm{T}}(\zeta)
$$


where $\zeta, \theta, \boldsymbol{a}_{\mathrm{tx}}(\zeta)$, and $\boldsymbol{a}_{\mathrm{rx}}(\theta)$ denote the Direction of Departure (DOD), Direction of Arrival (DOA), array steering response at transmitter and at receiver respectively. It is assumed that Uniform Linear Array (ULA) is employed at the transceiver, such that $\boldsymbol{a}_{\mathrm{tx}}^{\mathrm{H}}(\zeta) \boldsymbol{a}_{\mathrm{tx}}(\zeta)=M$ and $\boldsymbol{a}_{\mathrm{rx}}^{\mathrm{H}}(\theta) \boldsymbol{a}_{\mathrm{rx}}(\theta)=$ $N$. Moreover,

$$
\kappa=2 \pi F_{D} T_{s} \cos \psi
$$

where $F_{D}, T_{s}$, and $\psi$ describe the maximum doppler frequency, symbol period, and elevation angle between the transmitter and receiver respectively, hence, $\theta+\psi=90^{\circ}$. The LOS is doppler shifted by $F_{D} \cos \psi$, whereas $F_{D} T_{s}$ is regarded as normalized doppler. The phase shift $\phi$ is uniform over $(-\pi, \pi]$.

The time and spatial domain of the NLOS autocorrelation matrix is assumed independent [3]. Thus, assuming a first order autoregressive (AR) process with i.i.d. rayleigh elements [5], for any $l \leq n, n \geq 1$

$$
\boldsymbol{H}_{\mathrm{NLOS}}[n]=\rho^{n-l} \boldsymbol{H}_{\mathrm{NLOS}}[l]+\sum_{k=0}^{n-l-1} \rho^{k} \boldsymbol{R}[n-k]
$$

where $\boldsymbol{R}[n] \in \mathbb{C}^{N, M}$ is a random process with i.i.d. gaussian distribution and variance $\sigma_{r}^{2}$, while $\rho$ is the first order AR coefficient expressed as $\rho=$ $J_{0}\left(2 \pi F_{D} T_{s}\right)[5,6]$ with $J_{0}$ is the zero order Bessel function of the first kind.

\section{LS channel estimator and tracking performance}

At the receiver, channel estimation is performed by sending training sequence during the training phase, the result of which is used for symbol detection during the data phase. As such, only the training phase is considered here.

By the LS principle, the MIMO channel estimation algorithm minimizes the cost function [5]

$$
\sum_{i=1}^{n} \gamma^{n-i}\|\boldsymbol{y}[i]-\boldsymbol{H}[n] \boldsymbol{x}[i]\|^{2}
$$

where $\gamma$ is the forgetting factor. The estimated channel becomes

$$
\hat{\boldsymbol{H}}[n]=\left(\sum_{i=1}^{n} \gamma^{n-i} \boldsymbol{y}[i] \boldsymbol{x}^{\mathrm{H}}[i]\right)\left(\sum_{i=1}^{n} \gamma^{n-i} \boldsymbol{x}[i] \boldsymbol{x}^{\mathrm{H}}[i]\right)^{-1}
$$

The estimator is implemented with the recursive LS (RLS) algorithm. Defining the channel estimation error as

$$
\boldsymbol{\xi}[n]=\hat{\boldsymbol{H}}[n]-\boldsymbol{H}[n],
$$

the channel tracking MSE is determined as [5]

$$
\sigma_{\boldsymbol{H}}^{2}[n]=\frac{1}{M N} \operatorname{Tr}\left(E\left\{\boldsymbol{\xi}[n]^{\mathrm{H}} \boldsymbol{\xi}[n]\right\}\right)
$$

where $\operatorname{Tr}($.$) and E\{$.$\} are regarded matrix trace and expectation respectively.$ The cross correlation of the rician channel matrix at different time index emerges from

$$
\begin{aligned}
& E_{\boldsymbol{H}}\left\{(\boldsymbol{H}[l]-\boldsymbol{H}[n])^{\mathrm{H}}(\boldsymbol{H}[i]-\boldsymbol{H}[n])\right\}=N(1-\beta)\left(\rho^{|i-l|}+1-\rho^{n-l}-\rho^{n-i}\right) \boldsymbol{I} \\
& +N \beta\left(e^{j \kappa(i-l)}+1-e^{j \kappa(n-l)}-e^{-j \kappa(n-i)}\right) \boldsymbol{a}_{\mathrm{tx}}^{*}(\zeta) \boldsymbol{a}_{\mathrm{tx}}^{\mathrm{T}}(\zeta)
\end{aligned}
$$


where $E_{\boldsymbol{H}}\{\}$ denotes expectation w.r.t. matrix $\boldsymbol{H}$. By using Eq. (10), [5, Eq. (29)], and noticing that only the matrix trace is required, in rician fading

$$
\begin{aligned}
& E\left\{\boldsymbol{\xi}[n]^{\mathrm{H}} \boldsymbol{\xi}[n]\right\} \\
& =\boldsymbol{a}_{\mathrm{tx}}^{*}(\zeta) \boldsymbol{a}_{\mathrm{tx}}^{\mathrm{T}}(\zeta) \sum_{i=1}^{n} \sum_{l=1}^{n} \gamma^{2 n-l-i} N \beta\left(e^{j \kappa(i-l)}+1-e^{j \kappa(n-l)}-e^{-j \kappa(n-i)}\right) \\
& E_{\boldsymbol{x}}\left\{\boldsymbol{x}_{i} \boldsymbol{x}_{i}^{\mathrm{H}}\left(\sum_{q=1}^{n} \gamma^{n-q} \boldsymbol{x}_{q} \boldsymbol{x}_{q}^{\mathrm{H}}\right)^{-2} \boldsymbol{x}_{l} \boldsymbol{x}_{l}^{\mathrm{H}}\right\}+\sum_{i=1}^{n} \sum_{l=1}^{n} \gamma^{2 n-l-i} N(1-\beta) \\
& \left(\rho^{|i-l|}+1-\rho^{n-l}-\rho^{n-i}\right) E_{\boldsymbol{x}}\left\{\boldsymbol{x}_{l} \boldsymbol{x}_{l}^{\mathrm{H}} \boldsymbol{x}_{i} \boldsymbol{x}_{i}^{\mathrm{H}}\left(\sum_{q=1}^{n} \gamma^{n-q} \boldsymbol{x}_{q} \boldsymbol{x}_{q}^{\mathrm{H}}\right)^{-2}\right\} \\
& +N \sigma_{v}^{2} \sum_{i=1}^{n} \gamma^{2 n-2 i} E_{\boldsymbol{x}}\left\{\left(\boldsymbol{x}_{i} \boldsymbol{x}_{i}^{\mathrm{H}}\right)\left(\sum_{q=1}^{n} \gamma^{n-q} \boldsymbol{x}_{q} \boldsymbol{x}_{q}^{\mathrm{H}}\right)^{-2}\right\}
\end{aligned}
$$

From Eq. (11), the first term and the factor $(1-\beta)$ of the second term differentiate the tracking MSE of rician fading to the rayleigh one derived in [5]. As a consequence, the statistical expectation matrix of the first term is not given yet. By numerical study, it is allowed to proceed with the following approximation

$$
E_{\boldsymbol{x}}\left\{\boldsymbol{x}_{i} \boldsymbol{x}_{i}^{\mathrm{H}}\left(\sum_{q=1}^{n} \gamma^{n-q} \boldsymbol{x}_{q} \boldsymbol{x}_{q}^{\mathrm{H}}\right)^{-2} \boldsymbol{x}_{l} \boldsymbol{x}_{l}^{\mathrm{H}}\right\} \approx E_{\boldsymbol{x}}\left\{\boldsymbol{x}_{l} \boldsymbol{x}_{l}^{\mathrm{H}} \boldsymbol{x}_{i} \boldsymbol{x}_{i}^{\mathrm{H}}\left(\sum_{q=1}^{n} \gamma^{n-q} \boldsymbol{x}_{q} \boldsymbol{x}_{q}^{\mathrm{H}}\right)^{-2}\right\}
$$

where equality holds for $M=1$. The approximation shows good agreement numerically as far as the diagonal values are concerned, which is actually sufficient as the off-diagonal elements magnitude are negligible. By invoking Eq. (12), the expectation matrix between the first and second term of Eq. (11), now, becomes equivalent. As this matrix is diagonal [5], and only the matrix trace is required in Eq. (9), the matrix $\boldsymbol{a}_{\mathrm{tx}}^{*}(\zeta) \boldsymbol{a}_{\mathrm{tx}}^{\mathrm{T}}(\zeta)$ in Eq. (11), is thus neglected. By algebra manipulation, it is defined

$$
\begin{aligned}
& f(A, B)=2 A \frac{c_{1}}{1-\gamma B}\left(\gamma B \frac{1-\gamma^{2 n}}{1-\gamma^{2}}-(\gamma B)^{n} \frac{1-\left(\gamma B^{-1}\right)^{n}}{1-\gamma B^{-1}}\right) \\
& -2 A \frac{c_{2}}{1-\gamma B}\left(\gamma B \frac{1-\gamma^{3 n}}{1-\gamma^{3}}-(\gamma B)^{n} \frac{1-\left(\gamma^{2} B^{-1}\right)^{n}}{1-\gamma^{2} B^{-1}}\right) \\
& -2 A \frac{c_{2}}{1-\gamma^{2} B}\left(\gamma^{2} B \frac{1-\gamma^{3 n}}{1-\gamma^{3}}-\left(\gamma^{2} B\right)^{n} \frac{1-\left(\gamma B^{-1}\right)^{n}}{1-\gamma B^{-1}}\right) \\
& +2 A \frac{1-(\gamma B)^{n}}{1-\gamma B}\left(c_{2} \frac{1-\gamma^{2 n}}{1-\gamma^{2}}-c_{1} \frac{1-\gamma^{n}}{1-\gamma}\right) \\
& +2 A \frac{1-\left(\gamma^{2} B\right)^{n}}{1-\gamma^{2} B}\left(c_{2} \frac{1-\gamma^{n}}{1-\gamma}-c_{1}(M-1)\right)+2 A c_{2}(M-2) \frac{1-\left(\gamma^{3} B\right)^{n}}{1-\gamma^{3} B}
\end{aligned}
$$

which leads to the channel tracking MSE

$$
\begin{aligned}
& \sigma_{\boldsymbol{H}}^{2}[n]=c_{1} \frac{\left(1-\gamma^{n}\right)^{2}}{(1-\gamma)^{2}}+c_{1}\left(2 M-1+\sigma_{v}^{2}\right) \frac{1-\gamma^{2 n}}{1-\gamma^{2}}-2 c_{2}\left(\frac{1-\gamma^{2 n}}{1-\gamma^{2}}\right)\left(\frac{1-\gamma^{n}}{1-\gamma}\right) \\
& -c_{2}\left(2 M-2+\sigma_{v}^{2}\right) \frac{1-\gamma^{3 n}}{1-\gamma^{3}}+\operatorname{Re}\left\{f\left(A=\beta, B=e^{j \kappa}\right)\right\}+f(A=1-\beta, B=\rho)
\end{aligned}
$$


where $\operatorname{Re}\{$.$\} denotes the real part of a value, whereas c_{1}$ and $c_{2}$ are constants found in [5, Eq. (47)] and [5, Eq. (48)] respectively. The closed-form tracking MSE is generalized to rician fading, since setting $\beta=0$ in Eq. (14) leads to the rayleigh solution of [5, Eq. (53)] though with slight discrepancies ${ }^{1}$.

Although the approach taken follows closely [5], the result of Eq. (14) is not a straightforward extension of [5]. The fact that the MSE end result due to the LOS channel looks proportional to its NLOS counterpart, is not possible if we hadn't made the approximation in Eq. (12), which turned out to have satisfied the solution from [5] numerically. Furthermore, a lengthy algebra manipulation is involved in order to deal with the complex term $e^{j \kappa}$, producing in an additional Re\{.\} prior to the MSE contributed by the LOS channel.

\section{Analytical and simulation results}

Due to the assumed unit power of elements in $\boldsymbol{H}[n], \mathrm{SNR}=\frac{1}{\sigma_{v}^{2}}$. The simulation assumes QPSK modulation, $\mathrm{SNR}=10 \mathrm{~dB}, F_{D} T_{s}=0.01$, and averaging over 10000 independent realizations. The $K$ factor and elevation angle variations $\{\psi, K\}$ are chosen as $\left\{10^{\circ}, 0 \mathrm{~dB}\right\},\left\{60^{\circ}, 10 \mathrm{~dB}\right\}$, and $\left\{90^{\circ}, 20 \mathrm{~dB}\right\}[1]$.

The simulation and analytical results of tracking MSE versus training sequence index on MIMO, with $M=4$, and SIMO systems are illustrated in Fig. 1. Firstly, the description is given for MIMO system. The rayleigh curve with $\gamma=0.9$ is identical to [5, Fig. 5]. On the one hand, a very good agreement is found for both MIMO rayleigh and rician with $\gamma=0.9$, except on the initial starting point due to singularity of the autocorrelation matrix for $n<M$ [5]. On the other hand, validation of the tracking MSE is shown to already yield slight disparity for $\gamma=0.75$. Even in [5], the analytical expression of the second and third expectation matrices of Eq. (11) are only approximations. Thus, it is found that if $M \geq 2$, a higher minimum $\gamma$ is

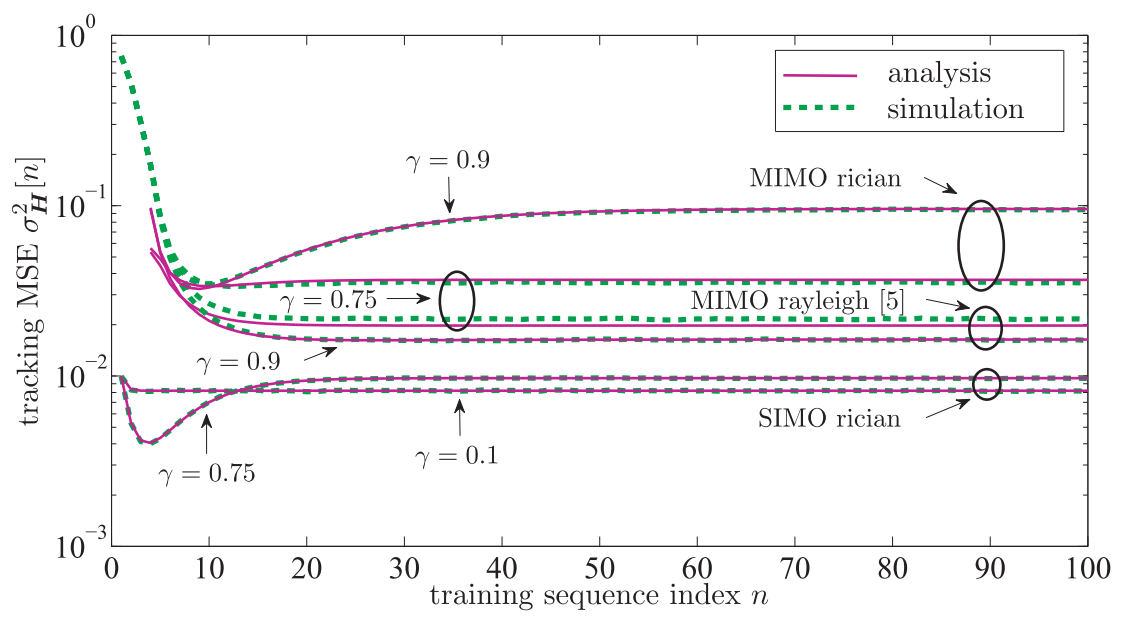

Fig. 1. Analytical and simulated tracking $\mathrm{MSE}, F_{D} T_{s}=$ 0.01; MIMO: $M=4$, SNR $=10 \mathrm{~dB}$; SIMO: $\mathrm{SNR}=20 \mathrm{~dB}$; rician: $\{\psi, K\}=\left\{60^{\circ}, 10 \mathrm{~dB}\right\}$ 


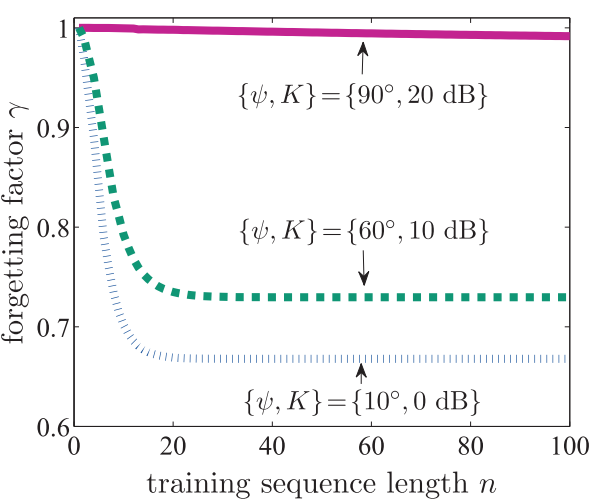

(a) $\gamma$ vs. training sequence length

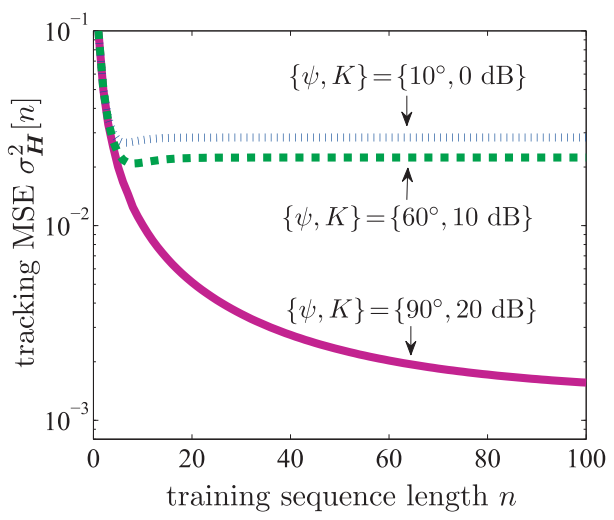

(b) $\sigma_{\boldsymbol{H}}^{2}$ vs. training sequence length

Fig. 2. Forgetting factor and its respective MSE obtained from $\frac{\partial\left(\sigma_{\boldsymbol{H}}^{2}[n]\right)}{\partial \gamma}=0 ; M=1, \mathrm{SNR}=10 \mathrm{~dB}, F_{D} T_{s}=$ 0.01

necessary as $M$ increases, otherwise, $\sigma_{\boldsymbol{H}}^{2}[n]<0$. Secondly, the explanation for SIMO system is presented, yet according to Fig. 1. The SNR is set higher to $20 \mathrm{~dB}$ for presentation clarity. In contrast to MIMO, a completely verified result is found on SIMO system for any $\gamma$, since the analytical solution of all expectation terms in Eq. (11) are exact. Therefore, the closed-form tracking MSE are exact on SIMO, whereas on MIMO, a very good match is found for some restricted $\gamma$ values.

It is of interest to see the impact of $\{\psi, K\}$ variations on the tracking performance by evaluating $\frac{\partial\left(\sigma_{\boldsymbol{H}}^{2}[n]\right)}{\partial \gamma}=0$. The values of $\gamma$, and consequently, $\sigma_{\boldsymbol{H}}^{2}[n]$, versus length of training sequence are depicted in Fig. $2(\mathrm{a})$ and (b) respectively. Decreasing $\psi$ from $90^{\circ}$ to $10^{\circ}$ leads to a faster time varying phase of the LOS component. Accordingly, as depicted in Fig. 2 (a), a lower $\gamma$ is demanded to ignore the contribution of old error signals quicker. For the $\{\psi, K\}=\left\{90^{\circ}, 20 \mathrm{~dB}\right\}$ case, the disturbance is practically AWGN, thus, $\gamma$ remains $\approx 1$ in order to average the noise power proportionally to the amount of received snapshots. If the mobile travels from $90^{\circ}$ to $10^{\circ}$ and capacity is of concerned, the training length is set fix in Fig. 2 (b). In this case, limiting the training to 10 symbols, the MSE is almost tripled. Hence, a higher tracking loss is introduced as the mobile travels to a lower elevation angle.

\section{Conclusion}

The tracking MSE of LS MIMO channel estimation in rician flat fading channel is derived in closed-form. The analytical result shows an exact match to simulation on SIMO system, whereas on MIMO, a very good match is found for some restricted forgetting factor values. This restriction is not yet mentioned in [5]. As far as the LOS component is concerned, the existence of elevation angle not equal to $90^{\circ}$ yet generates doppler shifted LOS, thus, a reduced forgetting factor and a higher tracking loss are produced as the mobile travels to a lower elevation angle. 